\title{
A hybrid Fermi-Ulam-bouncer model
}

\author{
Edson D. Leonel and P.V.E. McClintock \\ Department of Physics, Lancaster University, Lancaster, LA1 4YB, United Kingdom
}

\begin{abstract}
Some dynamical and chaotic properties are studied for a classical particle bouncing between two rigid walls, of which one is fixed and the other moves in time, in the presence of an external field. The system is a hybrid, behaving not as a purely Fermi-Ulam model, nor as a bouncer, but as a combination of the two. We consider two different kinds of motion of the moving wall: (i) periodic; and (ii) random. The dynamics of the model is studied via a two-dimensional nonlinear area-preserving map. We confirm that, for periodic oscillations, our model recovers the well-known results of the Fermi-Ulam model in the limit of zero external field. For intense external fields, we establish the range of control parameters values within which invariant spanning curves are observed below the chaotic sea in the low energy domain. We characterise this chaotic low energy region in terms of Lyapunov exponents. We also show that the velocity of the particle, and hence also its kinetic energy, grow according to a power law when the wall moves randomly, yielding clear evidence of Fermi acceleration.
\end{abstract}

\section{Introduction}

A special class of one-dimensional time-dependent systems exhaustively investigated in recent years are those related to the Fermi model. The latter system was originally proposed by Enrico Fermi [1] in order to study cosmic rays. It provides a mechanism through which charged particles can be accelerated by collisions with time-dependent magnetic fields. The model was later studied in different versions and using different approaches $[2,3,4,5,6,7,9,8,10]$. One of them consists in considering the dynamics of a classical particle bouncing between two rigid walls, one of which is fixed and the other one moves in time, known as the Fermi-Ulam model. The main result for periodic oscillation is that the phase space presents KAM islands surrounded by a chaotic sea. Unlimited energy growth (the condition for observing Fermi acceleration) is not, however, observed because the phase space exhibits a set of invariant spanning curves [11]. An alternative version of this model, proposed by Pustylnikov [12], is often referred to as a bouncer. It consists of a classical particle falling in a constant gravitational field, on a moving platform. The most important property of this system is that, in contradistinction to the Fermi-Ulam model, depending on both the initial conditions and control parameters there is no bound to the energy gained by the bouncing particle. This special difference between the models was clarified by Lichtenberg et al [13]. The quantum versions corresponding to both the bouncer and Fermi-Ulam models have also been studied $[14,15,16,17,18]$. 
The special interest attached to studying these one-dimensional classical systems is that they are completely integrable for zero external time-dependent forcing, but nonintegrable when the external forcing is switched on, and they allow direct comparisons of theoretical predictions with experimental results [19, 20, 21]. Such systems present a very rich phase space structure. Depending on the values chosen for the control parameters, and on the initial conditions, both periodic, quasi-periodic and chaotic behaviour can be observed. However, this kind of behaviour seems to be generic for no degenerated Hamiltonian systems and could be observed for time dependent potentials [22, 23, 24, 25, 26, 27], mesoscopic systems [28, 29], waveguides [30] and also for billiards with static boundaries [31, 32, 33]. Moreover, considering time-dependent boundaries for such billiards, the scenario can be quite different. In this sense, regular dynamics for a fixed boundary implies a bound to the energy gained by the bouncing particle, but the chaotic dynamics of a billiard with a fixed boundary is a sufficient condition for Fermi acceleration in the system when a boundary perturbation is introduced. A discussion of this interesting question, together with specific examples, can be found in Ref. [34].

In this paper we study a hybrid model that behaves, not as purely Fermi-Ulam model, nor as a bouncer, but as a combination of both. It consists of a time-dependent system where a classical particle is confined between two rigid walls in the presence of a gravitational field. One wall is fixed and the other one moves in time. We will consider in different sections two different kind of movement, namely: (i) periodic and (ii) random. For the periodic perturbation, the system is described by a two-dimensional nonlinear area-preserving map with two relevant control parameters. We will show that fixed points and periodic orbits go over smoothly into orbits of the Fermi-Ulam model as the external field goes to zero. We also show that the phase space presents a hierarchy of behaviours. It is possible to observe KAM islands surrounded by a chaotic sea that is limited by one invariant spanning curve. Considering an intense external field, we show that this system presents a set of invariant spanning curves located below the chaotic sea, indicating that the system then behaves mainly as a bouncer. If the wall moves randomly, the system exhibits the phenomenon of Fermi acceleration, which can be interpreted as evidence of the particle having unlimited energy gain.

This paper is organised as follows. In Sec. II we discuss in full detail all the steps used to derive the map describing the dynamics of this system. We construct both complete and simplified versions [35] of the problem. We also discuss briefly some results and properties of both the Fermi-Ulam and bouncer models that are useful for comparisons with results of our model. In Sec. III, we establish the connections between our hybrid model and the Fermi-Ulam model, and we discuss the features that emerge for the case of intense external fields. Sec. IV presents our numerical results for positive Lyapunov exponents, for both the complete and simplified models. In Sec. V we discuss the results obtained for the stochastic version of model, and Sec. VI summarises our results and presents our conclusions. 


\section{The model with periodic oscillations}

The model consists basically of a classical particle of mass $m$, subject to a constant gravitational field $g$, bouncing between two rigid walls. One of them is fixed at $y=l$ and the other one moves periodically in time according to the equation $y_{w}(t)=\epsilon^{\prime} \cos (\omega t)$. The parameter $\epsilon^{\prime}$ represents the amplitude of oscillation and $\omega$ is the angular frequency. Fig. 1 illustrates the model. We will study this problem in two different versions: (i) complete; and (ii) simplified. In the complete version, the model includes explicitly the movement of the oscillating wall. The instant of time at which each collision occurs is obtained numerically by solution of a transcendental equation. However, we can introduce a very useful and more simple version to this problem that allows easier analytical treatment: the simplified version. For the simplified version [35], we suppose that both walls are fixed but that, when the particle collides with the wall located at $y=0$, it exchanges momentum as if the wall was moving. This simplification forces us to introduce a strategy to avoid the successive collisions that occur only in the low energy domain for the complete model. At the same time, this version brings the advantage of allowing us to speed up our numerical simulations. The results obtained for a weak gravitational field and high energy are qualitatively almost the same as for the complete model, but differ for intense external fields or for low energy. However, in the simplified version, it is useful and much easier to identify generic behaviours that can immediately be sought in the corresponding complete version. We will observe that the quantitative results, in particular the positive Lyapunov exponent, are different in magnitude for the two versions of the model. Such differences are due to a few crucial intrinsic differences between the systems. We will describe the dynamics of both systems using a two dimensional map $T$ that gives us the corresponding pair of velocity $v_{n}$ and time $t_{n}$ values immediately after the collision with the oscillating wall, i.e. $\left(v_{n+1}, t_{n+1}\right)=T\left(v_{n}, t_{n}\right)$.

\subsection{The complete model}

Let us now begin to construct the map. Suppose that, after suffering a collision with the moving wall at a time $t=t_{n}$ at the position $y_{w}=\epsilon^{\prime} \cos \left(\omega t_{n}\right)$, the velocity of the particle is $v=v_{n}$. Depending on both the velocity $v_{n}$ and the time $t_{n}$, the two different kinds of behaviour that may occur are:

(a) The particle again collides with the moving wall before exiting the collision region. It is characterised as a successive collision.

(b) The particle exits the collision region without suffering any further collision.

The collision area is defined as the region in real space within which it is possible for the particle to suffer a collision with the moving wall, and it is given by $y \in\left[-\epsilon^{\prime}, \epsilon^{\prime}\right]$, as can be seen in Fig. 1. 


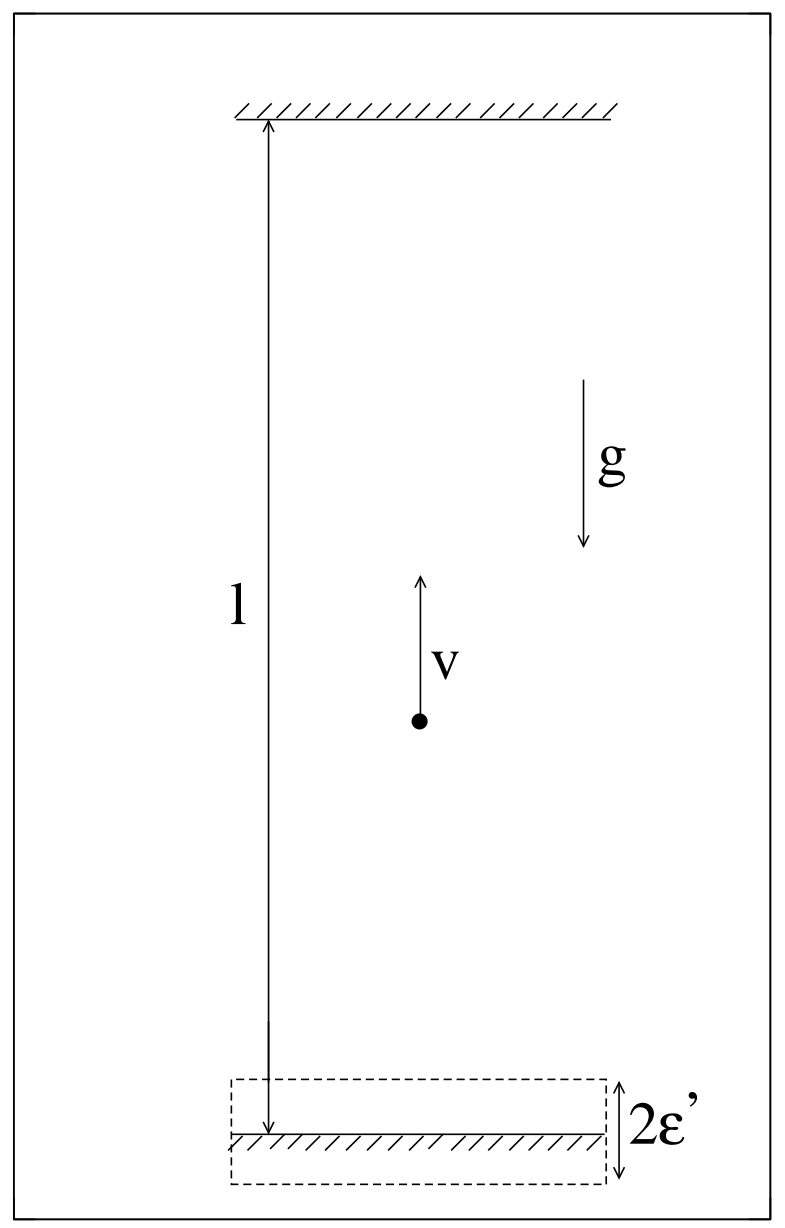

Figure 1. Sketch of the model with constant external field.

Considering first case (a), the instant of time at which the particle collides with the moving wall is obtained from the condition $y_{p}\left(t_{c}\right)=y_{w}\left(t_{c}\right)$, where $y_{p}\left(t_{c}\right)$ gives the position of the particle at time $t_{c}$. This condition leads to the transcendental equation

$$
g\left(t_{c}\right)=\epsilon^{\prime} \cos \left[\omega\left(t_{n}+t_{c}\right)\right]-\epsilon^{\prime} \cos \left(\omega t_{n}\right)-v_{n} t_{c}+\frac{1}{2} g t_{c}^{2} .
$$

Here, $t_{c}$ is the smallest root of $g\left(t_{c}\right)$ for $t_{c} \in(0,2 \pi / \omega]$. The time $t_{c}=0$ is excluded because it is a fixed point of $g\left(t_{c}\right)$. After the collision, the new time is given by $t_{n+1}=t_{n}+t_{c}$. The new velocity is obtained by requiring conservation of energy and momentum in the frame of reference of the moving wall (in which it is instantaneously at rest) and is given by $v_{n+1}=-v_{n}+g t_{c}-2 \omega \epsilon^{\prime} \sin \left(\omega t_{n+1}\right)$. The velocity of the moving wall, $v_{w}(t)$ is obtained using $\frac{d}{d t} y_{w}(t)=-\omega \epsilon^{\prime} \sin (\omega t)$. After that, we can write the map 
for successive collisions, $T_{s}$ as

$$
T_{s}:\left\{\begin{array}{l}
v_{n+1}=-v_{n}+g t_{c}-2 \omega \epsilon^{\prime} \sin \left(\omega t_{n+1}\right) \\
t_{n+1}=t_{n}+t_{c}
\end{array},\right.
$$

where $t_{c}$ is obtained numerically from $g\left(t_{c}\right)$.

If $g\left(t_{c}\right)$ does not have a solution in the interval $t_{c} \in(0,2 \pi / \omega]$, we can conclude that the particle leaves the collision area without suffering a successive collision. Case (b) then applies, and there are two new different kinds of behaviour that may occur:

$\left(b_{1}\right)$ The particle does not reach and collide with the fixed wall located at $y=l$, but returns in the downward direction due to the gravitational field alone.

$\left(b_{2}\right)$ The particle collides with the wall at $y=l$. It suffers a reversal of its momentum and returns downwards.

The basic condition for the particle suffering a collision with the fixed wall located at $y=l$ is $v_{n}>\sqrt{2 g\left[l-\epsilon^{\prime} \cos \left(\omega t_{n}\right)\right]}$. Otherwise, the particle returns without colliding with the fixed wall.

Let us first study case $\left(\mathrm{b}_{1}\right)$, i.e. $v_{n} \leq \sqrt{2 g\left[l-\epsilon^{\prime} \cos \left(\omega t_{n}\right)\right]}$. The time during which the particle rises and decelerates before its velocity falls transiently to zero is $t_{u}=v_{n} / g$. In this time, the particle reach a maximum height, $y_{\max }=\epsilon^{\prime} \cos \left(\omega t_{n}\right)+\frac{1}{2} \frac{v_{n}^{2}}{g}$. The particle is then accelerated downwards by the external gravitational field until it reaches the entrance to the collision area $y=\epsilon^{\prime}$. The time that the particle spends on this part of its trajectory is $t_{d}=\left(\sqrt{v_{n}^{2}+2 g\left[\epsilon^{\prime} \cos \left(\omega t_{n}\right)-\epsilon^{\prime}\right]}\right) / g$. At the entrance of the collision area, $y=\epsilon^{\prime}$, the velocity of the particle is

$$
v_{e}=-\sqrt{v_{n}^{2}+2 g\left[\epsilon^{\prime} \cos \left(\omega t_{n}\right)-\epsilon^{\prime}\right]} \text {. }
$$

After the particle enters this region, the instant of time at which the collision occurs is obtained from the condition $y_{p}\left(t_{c}\right)=y_{w}\left(t_{c}\right)$, giving rise to the transcendental equation:

$$
f\left(t_{c}\right)=\epsilon^{\prime} \cos \left[\omega\left(t_{n}+t_{u}+t_{d}+t_{c}\right)\right]-\epsilon^{\prime}-v_{e} t_{c}+\frac{1}{2} g t_{c}^{2} .
$$

Here, $t_{c}$ is the smallest root of the function $f\left(t_{c}\right)$ in the interval $t_{c} \in[0,2 \pi / \omega]$. We can now write the map $T_{n s}$ as

$$
T_{n s}:\left\{\begin{array}{l}
v_{n+1}=-v_{e}+g t_{c}-2 \omega \epsilon^{\prime} \sin \left(\omega t_{n+1}\right) \\
t_{n+1}=t_{n}+t_{u}+t_{d}+t_{c}
\end{array} .\right.
$$

We now consider situation $\left(\mathrm{b}_{2}\right)$, i.e. $v_{n}>\sqrt{2 g\left[l-\epsilon^{\prime} \cos \left(\omega t_{n}\right)\right]}$. The time that the particle spends on going up is $t_{u}=\left(v_{n}-\sqrt{v_{n}^{2}-2 g\left[l-\epsilon^{\prime} \cos \left(\omega t_{n}\right)\right]}\right) / g$. When the particle arrives at and collides with the fixed wall at $y=l$, its velocity is given by $v=\sqrt{v_{n}^{2}-2 g\left[l-\epsilon^{\prime} \cos \left(\omega t_{n}\right)\right]}$. The time it spends on going down before it reaches the entrance to the collision area is given by $t_{d}=\left(\sqrt{v_{n}^{2}+2 g\left[\epsilon^{\prime} \cos \left(\omega t_{n}\right)-\epsilon^{\prime}\right]}\right) / g-$ $\left(\sqrt{v_{n}^{2}-2 g\left[l-\epsilon^{\prime} \cos \left(\omega t_{n}\right)\right]}\right) / g$. The velocity of the particle immediately before entering the collision area $v_{e}$ is still described by Eq. (3). The instant of time at which it suffers a collision with the moving wall is obtained by solution of $f\left(t_{c}\right)$. Again, $t_{c}$ is the smallest 
value of $t_{c} \in[0,2 \pi / \omega]$. After the collision, the new velocity and the new time are again given by applying the map $T_{n s}$.

It is easy to see that, in the form (2) and (5) in which the map $T$ was constructed, there is an excessive number of control parameters, 4 in total, namely $\epsilon^{\prime}, \omega, l$ and $g$. We now define dimensionless and much more appropriate variables in order to reduce the effective and relevant control parameters to just 2. The first change is to measure time in terms of the period of oscillation of the moving wall, $\phi_{n}=\omega t_{n}$. We can also define a new velocity as $V_{n}=v_{n} /(\omega l)$ so that the new amplitude of oscillation is given by $\epsilon=\epsilon^{\prime} / l$. Using the period of oscillation $\tau$, given by $\tau=2 \pi / \omega$, we are able to define a new variable $N_{c}=\tau_{c} / \tau$. Here, $\tau_{c}^{2}=2 l / g$ gives us the squared time that the particle spends in travelling the distance $l$ under the influence of the gravitational field $g$ starting with initial velocity $v=0$. In this way, $N_{c}$ gives us the number of oscillations that the moving wall completes in a time $\tau_{c}$.

Using these new variables, the map is written as

$$
T:\left\{\begin{array}{l}
V_{n+1}=V_{n}^{*}+\frac{\phi_{c}}{2 \pi^{2} N_{c}^{2}}-2 \epsilon \sin \left(\phi_{n+1}\right) \\
\phi_{n+1}=\phi_{n}+\Delta T_{n} \bmod 2 \pi
\end{array}\right.
$$

where $\Delta T_{n}$ and $V_{n}^{*}$ are given by different expressions that depend on the following conditions:

(i) Successive collision. $\Delta T_{n}=\phi_{c}$ and $V_{n}^{*}=-V_{n}$. Here $\phi_{c}$ is obtained as the smallest root of $G\left(\phi_{c}\right)$ in the interval $\phi_{c} \in(0,2 \pi]$ and $G\left(\phi_{c}\right)$ is given by

$$
G\left(\phi_{c}\right)=\epsilon \cos \left(\phi_{n}+\phi_{c}\right)-\epsilon \cos \left(\phi_{n}\right)+V_{n}^{*} \phi_{c}+\frac{\phi_{c}^{2}}{4 \pi^{2} N_{c}^{2}} .
$$

(ii) Indirect collision without reflection, $V_{n} \leq \frac{\sqrt{1-\epsilon \cos \left(\phi_{n}\right)}}{\pi N_{c}}$. This condition establishes an indirect collision without reflection at $y=1$. Then

$$
\begin{aligned}
\Delta T_{n} & =\phi_{u}+\phi_{d}+\phi_{c}, \\
V_{n}^{*} & =\sqrt{V_{n}^{2}+\frac{\epsilon}{\pi^{2} N_{c}^{2}}\left[\cos \left(\phi_{n}\right)-1\right] .}
\end{aligned}
$$

Here $\phi_{u}=2 \pi^{2} N_{c}^{2} V_{n}, \phi_{d}=2 \pi N_{c} \sqrt{\epsilon \cos \left(\phi_{n}\right)-\epsilon+\pi^{2} N_{c}^{2} V_{n}^{2}}$ and $\phi_{c}$ is obtained numerically as the smallest root of $F\left(\phi_{c}\right)$ in the interval $\phi_{c} \in[0,2 \pi]$, where $F\left(\phi_{c}\right)$ is given by

$$
F\left(\phi_{c}\right)=\epsilon \cos \left(\phi_{n}+\phi_{u}+\phi_{d}+\phi_{c}\right)-\epsilon+V_{n}^{*} \phi_{c}+\frac{\phi_{c}^{2}}{4 \pi^{2} N_{c}^{2}}
$$

(iii) Indirect collision with reflection, $V_{n}>\frac{\sqrt{1-\epsilon \cos \left(\phi_{n}\right)}}{\pi N_{c}}$. This condition establishes an indirect collision with reflection at $y=1$. The terms $\Delta T_{n}$ and $V_{n}^{*}$ are given by Eq. (8) and (9), as in the previous case. The only the difference is that here $\phi_{u}=2 \pi N_{c}\left[\pi N_{c} V_{n}-\sqrt{\pi^{2} N_{c}^{2} V_{n}^{2}-1+\epsilon \cos \left(\phi_{n}\right)}\right], \phi_{d}=$ $2 \pi N_{c}\left[\sqrt{\epsilon \cos \left(\phi_{n}\right)-\epsilon+\pi^{2} N_{c}^{2} V_{n}^{2}}-\sqrt{\epsilon \cos \left(\phi_{n}\right)-1+\pi^{2} N_{c}^{2} V_{n}^{2}}\right]$, and $\phi_{c}$ is obtained numerically as the smallest root of Eq. (10) under the same conditions as in the previous case. 


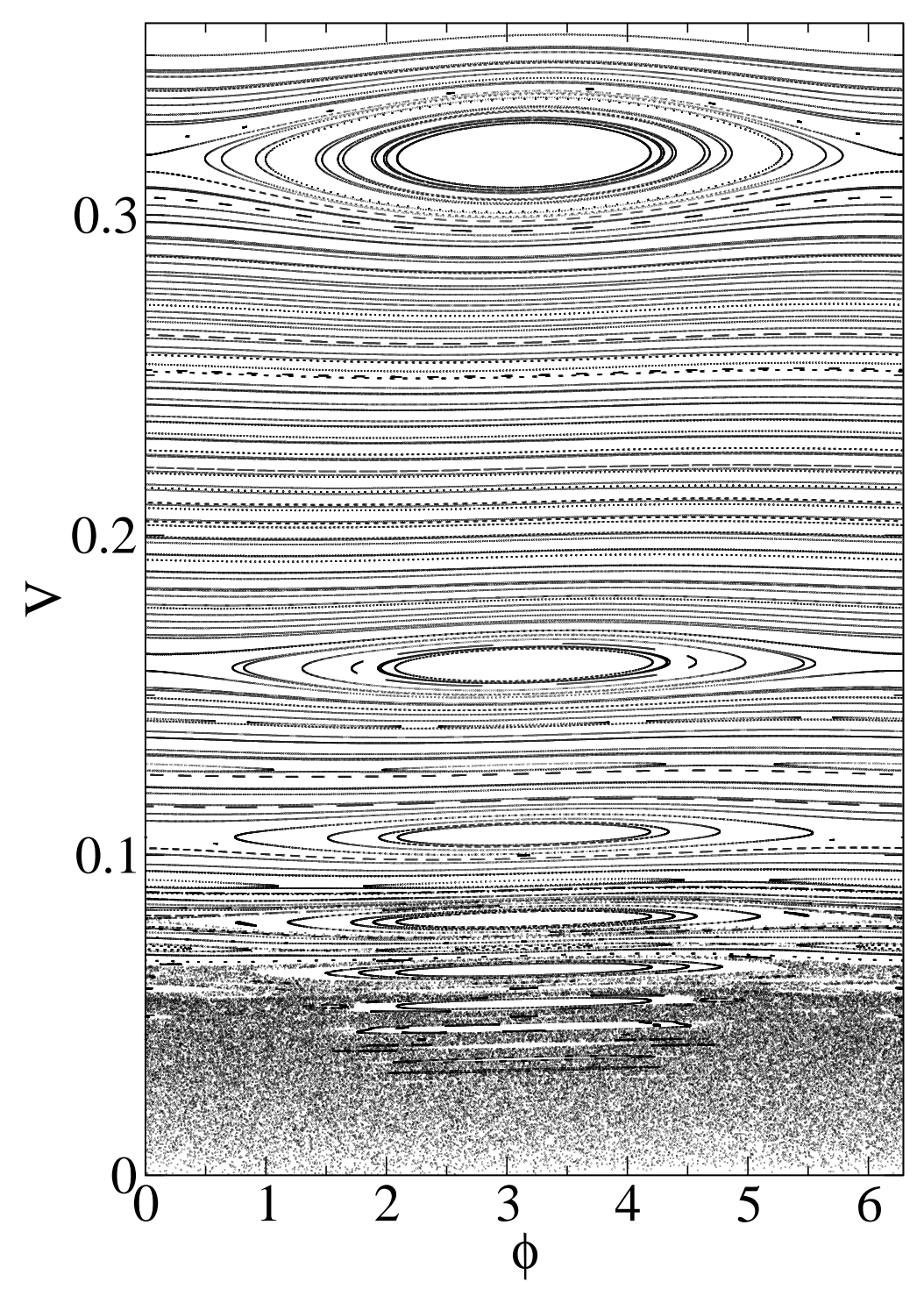

Figure 2. Phase space for the complete model. The control parameters used here were $\epsilon=10^{-3}$ and $N_{c}=28$.

After some tedious but necessary algebra, we can show that this map preserves the following measure on the phase space: $d \mu=[V+\epsilon \sin (\phi)] d V d \phi$. It is interesting that this measure $d \mu$ is the same as that obtained [10] for the Fermi-Ulam model itself. The same measure was also found for the breathing circle [36]. Note however that a diametrical orbit in the breathing circle necessarily recovers the results of the FermiUlam model.

A typical phase space for the complete model is shown in Fig. 2. The control parameters used were $\epsilon=1 \times 10^{-3}$ and $N_{c}=28$. For this combination of control parameters, a hierarchy of behaviours is immediately evident. In the low energy regime, the system shows a set of KAM islands that are surrounded by a chaotic sea. This 
chaotic sea, characterised by positive Lyapunov exponents, is limited by one invariant spanning curve. Above the chaotic sea, it is possible to observe KAM islands as well as small chaotic regions confined by two different invariant spanning curves. For yet higher values of energy, we can see basically invariant spanning curves. A characteristic feature of this model, quite unlike the Fermi-Ulam model, is that in the low energy regime for appropriate combinations of control parameters (especially for intense external fields), it is possible to observe a set of invariant spanning curves as well as stable fixed points. We will show that this kind of behaviour is property of the bouncer model. A more detailed discussion of the necessary conditions for such observations will be given in Sec. 3 .

We now describe briefly the tool used to characterise the chaotic low energy region: the Lyapunov exponent. It is well known as a practical tool that can quantify the average expansion or contraction rate for a small volume of initial conditions. One can say that a negative Lyapunov exponent implies a convergence of nearby initial conditions. An orbit that has this property is said to be regular (periodic or quasi-periodic). When the Lyapunov exponent is positive, however, it characterises an exponential divergence of initial conditions and it is related to chaotic motion. An orbit that is characterised by a positive Lyapunov exponent is referred to as chaotic. We use the algorithm of triangularisation [37] to evaluate the Lyapunov exponents. They are defined as

$$
\lambda_{j}=\lim _{N \rightarrow \infty} \frac{1}{N} \sum_{n=1}^{N} \ln \left|\Lambda_{j}^{n}\right|, \quad j=1,2,
$$

where $\Lambda_{j}^{n}$ are the corresponding eigenvalues of $D T^{N}=\prod_{n=1}^{N} J_{n}$. The matrix $J$ is the Jacobian matrix for the map $T$. As the map is area-preserving, the Lyapunov exponents obey the relationship that $\lambda_{1}=-\lambda_{2}$. Fig. 3(a) shows the convergence for 5 different initial conditions related to the chaotic sea, for the complete model. Each initial condition was iterated $5 \times 10^{8}$ times in order to produce a good convergence of the asymptotically positive Lyapunov exponent. The ensemble average of five different initial conditions gives us that $\bar{\lambda}=0.794 \pm 0.005$. The error represents the standard deviation of the five samples.

\subsection{The simplified model}

Let us now discuss the simplified model [35]. It consists basically in supposing that both walls are fixed but that, after each collision, the wall located at $y=0$ transfers momentum to the particle as if it was moving. It can immediately be seen that the transcendental equations $G\left(\phi_{c}\right)$ and $F\left(\phi_{c}\right)$ no longer need to be solved. Although this simplification brings the huge advantage of allowing very fast simulations, it also gives rise to a problem that we need to avoid. In the complete model, depending on the combination of both velocity and phase, it is possible for the particle, after suffering a collision with the moving wall, to suffer a second successive collision before exiting the collision area, as well as possibly having a negative velocity following the first such a collision. In the simplified model, non-positive velocities are forbidden because they are 
(a)

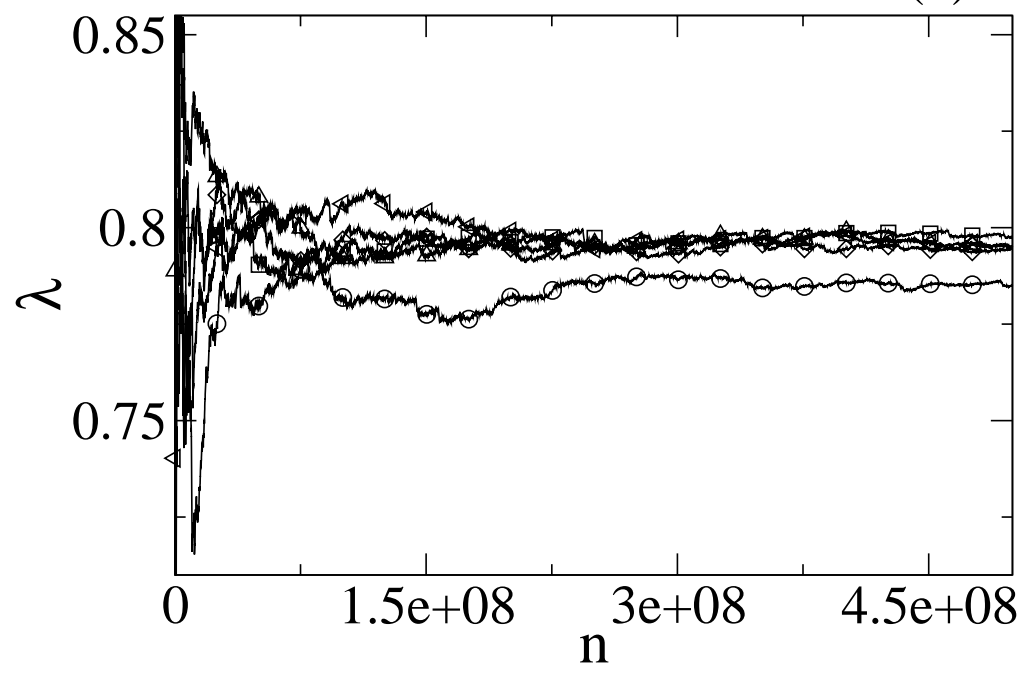

(b)

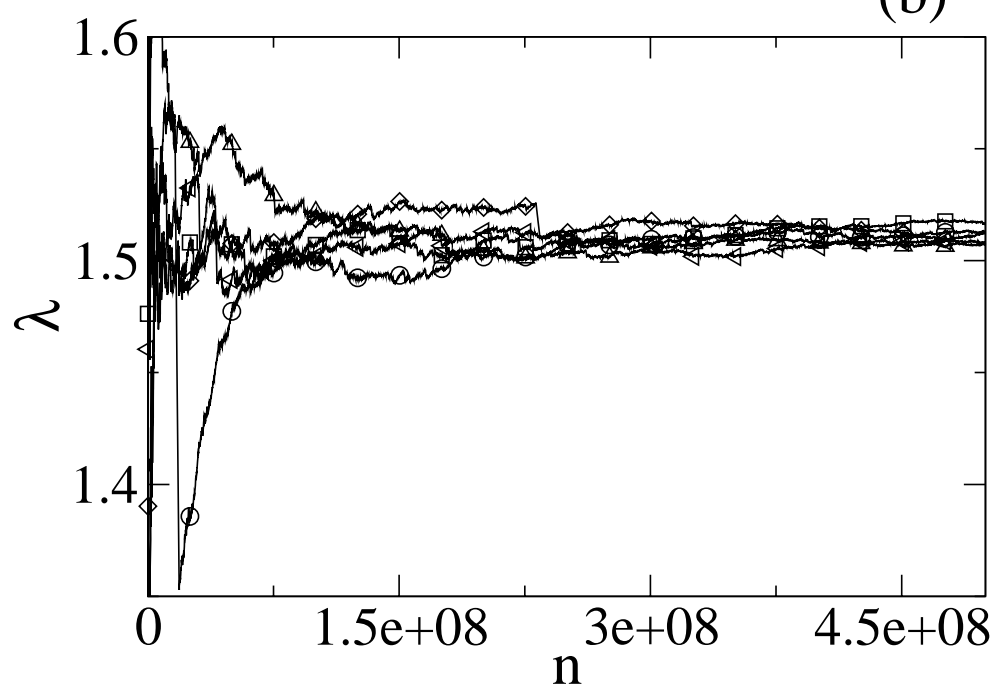

Figure 3. Time evolution from 5 different initial conditions related to the chaotic sea on: (a) the complete and (b) the simplified model. The control parameters used were $\epsilon=1 \times 10^{-3}$ and $N_{c}=28$.

equivalent to the particle travelling beyond the wall. In order to avoid such problems, if after the collision the particle has a negative velocity, we inject it back with the same modulus of velocity. Such a procedure is effected perfectly by use of a module function. Note that the velocity of the particle is reversed by the module function only if, after the collision, the particle remains travelling in the negative direction. The module function has no effect on the motion of the particle if it moves in the positive direction after the collision. We stress that this approximation is valid only for small values of $\epsilon$. In 
accordance with this discussion, the map for the simplified model is written as

$$
\left\{\begin{array}{l}
V_{n+1}=\left|V_{n}-2 \epsilon \sin \left(\phi_{n+1}\right)\right| \\
\phi_{n+1}=\phi_{n}+\Delta T_{n} \quad \bmod 2 \pi
\end{array},\right.
$$

where

$$
\begin{aligned}
& \Delta T_{n}=4 \pi^{2} N_{c}^{2}\left[V_{n}-\sqrt{V_{n}^{2}-\frac{1}{\pi^{2} N_{c}^{2}}}\right] \text { if } V_{n}>\frac{1}{\pi N_{c}} \\
& \Delta T_{n}=4 \pi^{2} N_{c}^{2} V_{n} \quad \text { if } V_{n} \leq \frac{1}{\pi N_{c}}
\end{aligned}
$$

This map is area-preserving because $\operatorname{det} J= \pm 1$. The phase space for the simplified version shows basically the same properties present in the complete model, i.e. it has a structure of KAM islands, a chaotic sea and invariant spanning curves. However, due to the module function, the velocity of the particle has the following constraint $\left[V_{n}-2 \epsilon \sin \left(\phi_{n+1}\right)\right]>0$. It is shown in Fig. 3(b) the time evolution of the positive Lyapunov exponent for 5 different initial conditions related to the chaotic sea. The asymptotic value is $1.509 \pm 0.006$. Here, the error of 0.006 represents the standard deviation of the five samples.

\subsection{Fermi-Ulam model}

Before considering the connection between the models, let us briefly discuss some properties of the Fermi-Ulam model. Considering $g=0$ on Fig. 1, and using the optimal variables, the map describing the dynamics of the simplified Fermi-Ulam model is given by

$$
\left\{\begin{array}{l}
V_{n+1}=\left|V_{n}-2 \epsilon \sin \left(\phi_{n+1}\right)\right| \\
\phi_{n+1}=\phi_{n}+\frac{2}{V_{n}} \quad \bmod 2 \pi
\end{array} .\right.
$$

The phase space for this system exhibits KAM islands surrounded by a chaotic sea that is limited by one invariant spanning curve in the low energy domain. For high energy, it shows basically a set of invariant spanning curves. As discussed in [10], the position of the first invariant spanning curve can be rescaled for different control parameters and connected to the Standard Map (SM) to appear for the same effective control parameter $\left(K_{\mathrm{FU}} \approx 0.971 \ldots\right)$ at which the SM undergoes a change of locally to globally stochastic behaviour [39]. Such scaling provides the explanation for the quasi-invariance of the positive Lyapunov exponent in the chaotic sea for this model [10]. (See also Ref. [7] for recent results of the simplified Fermi-Ulam model). Table I shows the classification of periodic orbits for the Fermi-Ulam model.

\subsection{Bouncer model}

In this section we will discuss a special transition observed in the bouncer model. For simplicity and to make it easier to compare some results of this model with those for our hybrid model, we will use the same variables to describe both models. To obtain the bouncer model, we just need to move out the fixed wall located at $y=l$ in Fig. 


\begin{tabular}{cccll}
\hline Period & \multicolumn{1}{c}{$\phi$} & $\phi$ & $\epsilon$ & Type \\
\hline 1 & $\frac{1}{j \pi}, j=1,2,3 \ldots$ & 0 & all & Hyperbolic \\
\hline 1 & $\frac{1}{j \pi}, j=1,2,3 \ldots$ & $\pi$ & $<\frac{1}{j^{2} \pi^{2}}$ & Elliptic \\
\hline 1 & $\frac{1}{j \pi}, j=1,2,3 \ldots$ & $\pi$ & $=\frac{1}{j^{2} \pi^{2}}$ & Parabolic \\
\hline 1 & $\frac{1}{j \pi}, j=1,2,3 \ldots$ & $\pi$ & $>\frac{1}{j^{2} \pi^{2}}$ & Hyperbolic \\
\hline 2 & $\frac{2}{j \pi}, j=1,3,5 \ldots$ & 0 and $\pi$ & $<\frac{2}{j^{2} \pi^{2}}$ & Elliptic \\
\hline 2 & $\frac{2}{j \pi}, j=1,3,5 \ldots$ & 0 and $\pi$ & $=\frac{2}{j^{2} \pi^{2}}$ & Parabolic \\
\hline 2 & $\frac{2}{j \pi}, j=1,3,5 \ldots$ & 0 and $\pi$ & $>\frac{2}{j^{2} \pi^{2}}$ & Hyperbolic \\
\hline
\end{tabular}

Table 1. Classification of periodic orbits of periods 1 and 2 for the simplified FermiUlam model.

1. In this way, the map that describes the dynamics of the simplified bouncer model is given by

$$
\left\{\begin{array}{l}
V_{n+1}=\left|V_{n}-2 \epsilon \sin \left(\phi_{n+1}\right)\right| \\
\phi_{n+1}=\phi_{n}+4 \pi^{2} N_{c}^{2} V_{n} \quad \bmod 2 \pi
\end{array} .\right.
$$

The term $4 \pi^{2} N_{c}^{2} V_{n}$ represents the time that the particle spends travelling between impacts. The special advantage of this system is that it allow the possibility of predicting analytically where the transition occurs between locally and globally stochastic behaviour [39]. To put the map (16) in the same form as the SM, we must firstly to define two variables. The first one is $I_{n}=4 \pi^{2} N_{c}^{2} V_{n}$ and the second one is $\theta_{n}=\phi_{n+1}+\pi$. In terms of these new variables, the map (16) may now be rewritten in the same form as the SM [3]

$$
\left\{\begin{array}{l}
I_{n+1}=I_{n}+K_{\mathrm{B}} \sin \left(\theta_{n}\right) \\
\theta_{n+1}=\theta_{n}+I_{n+1}
\end{array} .\right.
$$

The effective control parameter $K_{\mathrm{B}}=8 \pi^{2} \epsilon N_{c}^{2}$. However it is expected that, if $K_{\mathrm{B}} \geq$ $0.971 \ldots$, the SM will exhibit globally stochastic behaviour [39]. To characterise such a transition in the bouncer model in terms of both $N_{c}$ and $\epsilon$, we just need to evaluate the effective control parameter $K_{\mathrm{B}}$. Using the above condition, $8 \pi^{2} \epsilon N_{c}^{2}=K_{\mathrm{B}} \geq 0.971 \ldots$, whence

$$
N_{c} \geq \frac{1}{2 \pi} \sqrt{\frac{0.971 \ldots}{2 \epsilon}} .
$$

For $\epsilon=10^{-3}$, we thus obtain $N_{c} \geq 3.507 \ldots$ 


\section{The limits of $N_{c}$}

We will discuss in this section how the connection of the present model to the FermiUlam one can be established. It is well known that the parameter $N_{c}=\tau_{c} / \tau$ describes the intensity of the gravitational field. It effectively specifies the number of oscillations that the moving wall (in the complete model) executes during the time interval $\tau_{c}$. This tells us the length of time during which the particle travels a distance $l$ (in the original model), under a gravitational field $g$ after starting with an initial velocity $V=0$. We can conclude that a huge (small) value of $N_{c}$ implies motion in a weak (intense) gravitational field. In this way, it is clear: (i) that $N_{c} \rightarrow \infty$ implies $g \rightarrow 0$; and (ii) that $N_{c} \rightarrow 0$ implies $g \rightarrow \infty$.

\subsection{The limit $N_{c} \rightarrow \infty$}

Let us first discuss case (i). In this situation, it is supposed that the results of the Fermi-Ulam model should be recovered. For simplicity of calculation, we will use the simplified model although, with a bit more work, just the same procedure can be applied for the complete version too. The application of $N_{c} \rightarrow \infty$ excludes the Eq. (14) from the relation of $\Delta T_{n}$ on the simplified model because we are considering only non-negative velocities. We can now expand Eq. (13), obtaining

$$
\Delta T_{n}=\lim _{N_{c} \rightarrow \infty} 4 \pi^{2} N_{c}^{2} \times\left[V_{n}-\left(\sqrt{V_{n}^{2}}-\frac{1}{2 \pi^{2} \sqrt{V_{n}^{2}} N_{c}^{2}}\right)+\ldots\right]
$$

Considering that all the higher order terms disappear when the limit is applied, we can greatly simplify the last equation to

$$
\Delta T_{n}=\frac{2}{V_{n}}
$$

This is the equation that gives the length of time which a particle spends between collisions in the Fermi-Ulam model (see Sec. 2.3) and demonstrates that, in the present case, the Fermi-Ulam map is indeed recovered.

It is also interesting to characterise how the fixed points for our present model go over into the fixed points of the Fermi-Ulam model. It is well known that periodic orbits are obtained when the following conditions are satisfied: $V_{n+m}=V_{n}$ and $\phi_{n+m}=\phi_{n}$. Here, $m$ gives the periodicity of the orbit, so that one has period-one orbits with $m=1$, period-2 orbits with $m=2$, and so on. To characterise such an evolution, let us consider first the case $V_{n}>1 /\left(\pi N_{c}\right)$. The equations to be solved for the fixed points are

$$
\begin{aligned}
& \phi_{n+1}=\phi_{n}+4 \pi^{2} N_{c}^{2}\left[V_{n}-\sqrt{V_{n}^{2}-\frac{1}{\pi^{2} N_{c}^{2}}}\right]=\phi_{n}, \\
& V_{n+1}=V_{n}-2 \epsilon \sin \left(\phi_{n+1}\right)=V_{n} .
\end{aligned}
$$

Their solution gives us that

$$
V_{n}=\frac{j}{4 \pi N_{c}^{2}}+\frac{1}{j \pi} \quad \text { with } \quad j=1,2,3 \ldots \leq 2 N_{c}
$$


and $\phi_{n}=0$ or $\phi_{n}=\pi$. The fixed points $\left(V_{n}, \phi_{n}\right)=\left(\frac{j}{4 \pi N_{c}^{2}}+\frac{1}{j \pi}, 0\right)$ are hyperbolic for all values of $\epsilon$. The classification of fixed points $\left(V_{n}, \phi_{n}\right)=\left(\frac{j}{4 \pi N_{c}^{2}}+\frac{1}{j \pi}, \pi\right)$ is summarised in Table II. Comparing these results to those presented above in Table I, it is easy to see

\begin{tabular}{clll}
\hline$V_{n}$ & $\phi_{n}$ & $\epsilon$ & Type \\
\hline$\frac{j}{4 \pi N_{c}^{2}}+\frac{1}{j \pi}$ & 0 & all & Hyperbolic \\
\hline$\frac{j}{4 \pi N_{c}^{2}}+\frac{1}{j \pi}$ & $\pi$ & $<\frac{1}{j^{2} \pi^{2}}-\frac{1}{4 \pi^{2} N_{c}^{2}}$ & Elliptic \\
\hline$\frac{j}{4 \pi N_{c}^{2}}+\frac{1}{j \pi}$ & $\pi$ & $=\frac{1}{j^{2} \pi^{2}}-\frac{1}{4 \pi^{2} N_{c}^{2}}$ & Parabolic \\
\hline$\frac{j}{4 \pi N_{c}^{2}}+\frac{1}{j \pi}$ & $\pi$ & $>\frac{1}{j^{2} \pi^{2}}-\frac{1}{4 \pi^{2} N_{c}^{2}}$ & Hyperbolic \\
\hline
\end{tabular}

Table 2. Classification of the period-one orbits of the simplified model for $V_{n}>$ $1 /\left(\pi N_{c}\right)$.

that the expressions for both the fixed point $V_{n}$ and for the control parameter go over smoothly to those of the Fermi-Ulam model when the limit $N_{c} \rightarrow \infty$ is applied.

Moving now to consider the case $V_{n} \leq \frac{1}{\pi N_{c}}$, the conditions for finding the fixed points are

$$
\begin{aligned}
& \phi_{n+1}=\phi_{n}+4 \pi^{2} N_{c}^{2} V_{n}=\phi_{n}, \\
& V_{n+1}=V_{n}-2 \epsilon \sin \left(\phi_{n+1}\right)=V_{n} .
\end{aligned}
$$

Solving these conditions, we find that $V_{n}=\frac{j}{2 \pi N_{c}^{2}}$ with $j=1,2,3 \ldots \leq 2 N_{c}$ and $\phi_{n}=0$ or $\phi_{n}=\pi$. The fixed points $\left(V_{n}, \phi_{n}\right)=\left(\frac{j}{2 \pi N_{c}^{2}}, \pi\right)$ are hyperbolic for all values of $\epsilon$ whereas $\left(V_{n}, \phi_{n}\right)=\left(\frac{j}{2 \pi N_{c}^{2}}, 0\right)$ are elliptic for $\epsilon<\frac{1}{2 \pi^{2} N_{c}^{2}}$, parabolic for $\epsilon=\frac{1}{2 \pi^{2} N_{c}^{2}}$ and hyperbolic for $\epsilon>\frac{1}{2 \pi^{2} N_{c}^{2}}$. The same analysis for the period-two orbit yields

$$
\begin{aligned}
& \phi_{n+2}=\phi_{n}+8 \pi^{2} N_{c}^{2}\left[V_{n}-\epsilon \sin \left(\phi_{n}+4 \pi^{2} N_{c}^{2} V_{n}\right)\right]=\phi_{n}, \\
& V_{n+2}=V_{n}-2 \epsilon\left[\sin \left(\phi_{n}+4 \pi^{2} N_{c}^{2} V_{n}\right)+\sin \left(\phi_{n+2}\right)\right]=V_{n} .
\end{aligned}
$$

The solutions under such conditions give us that $V_{n+1}=V_{n}=\frac{j}{4 \pi N_{c}^{2}}$ with $j=$ $1,3,5 \ldots \leq 4 N_{c}, \phi_{n}=0$ and $\phi_{n+1}=\pi$. Such a period-two orbit is elliptic for $\epsilon<\frac{1}{4 \pi^{2} N_{c}^{2}}$, parabolic for $\epsilon=\frac{1}{4 \pi^{2} N_{c}^{2}}$ and hyperbolic for $\epsilon>\frac{1}{4 \pi^{2} N_{c}^{2}}$. All periodic orbits obtained for the condition $V_{n} \leq \frac{1}{\pi N_{c}}$ are the same that are present in the bouncer model. To be more precise, for the bouncer model there is not constraint to the values of $j$. In that model, $j$ can run from 1 to infinity. Such fixed points and periodic orbits do not exist on the Fermi-Ulam model; they are exclusively present in the bouncer model. Applying the limit $N_{c} \rightarrow \infty$, we see that both the period-one and period-two orbits disappear.

\subsection{The $N_{c}$ small values limit}

We will discuss in this section what happens in the limit $N_{c} \rightarrow 0$. It is obvious that both complete and simplified model are singular in this limit, in the sense that they present divergences of the velocity $(V \rightarrow \infty)$ to 
(a)

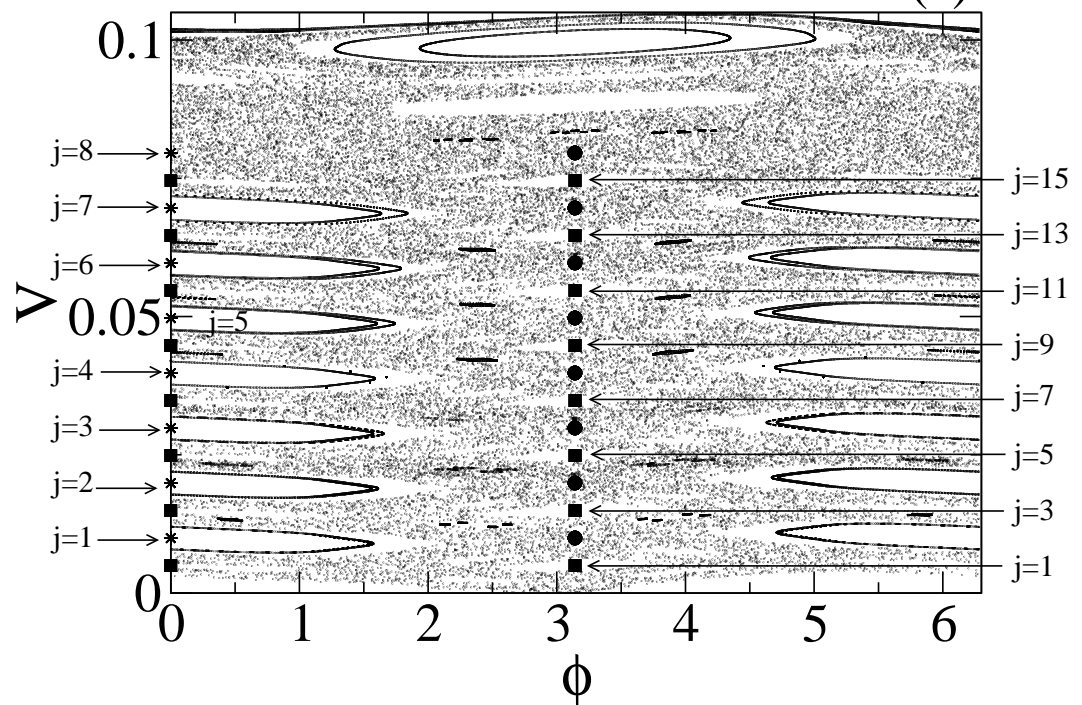

(b)

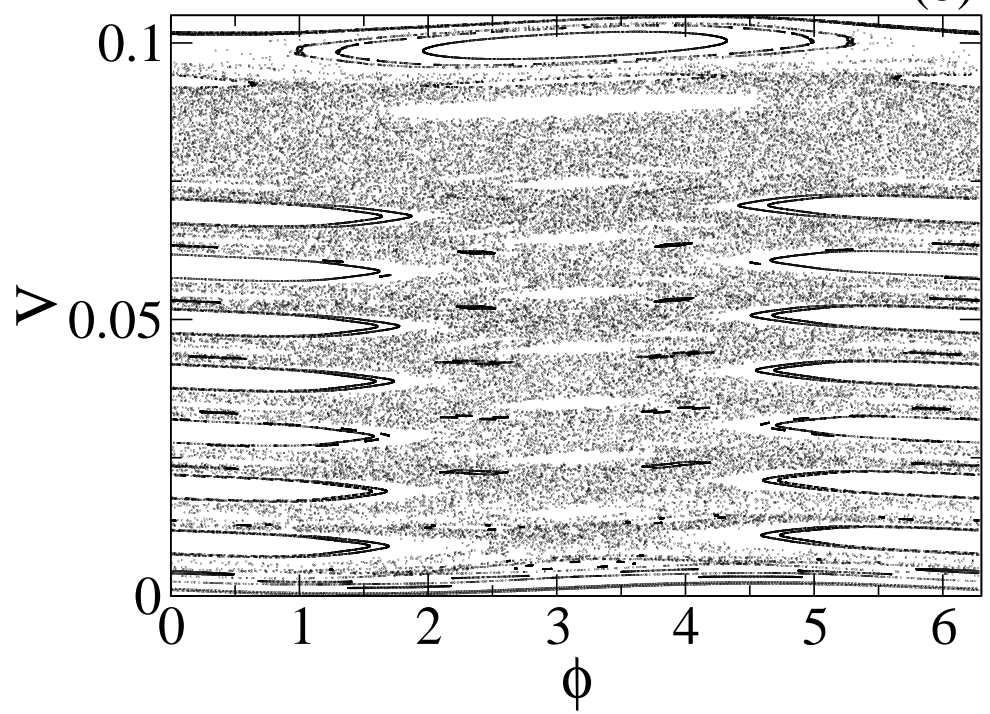

Figure 4. Phase space at low energy for both the (a) simplified and (b) complete models. In (a) the corresponding period-one and period-two orbits for $V \leq 1 / \pi N_{c}$ are shown. The star indicates elliptic fixed points, the filled circles give the corresponding hyperbolic fixed points with the same label as the star just above, and the squares illustrate the period-two orbits. (b) shows invariant spanning curves below the chaotic sea for the complete model. The control parameters used here were $\epsilon=10^{-3}$ and $N_{c}=4$.

the fixed points. Rather than applying the limit $N_{c} \rightarrow 0$ directly, we will discuss some results for small values of $N_{c}$. We saw in Fig. 2 that the combination of control parameters used there yields a phase space exhibiting KAM islands surrounded by a chaotic sea in the low energy regime. That chaotic sea is limited by an invariant spanning curve. The particular new result for this model is that, depending on the control parameter values, a set of invariant spanning curves located below the chaotic 

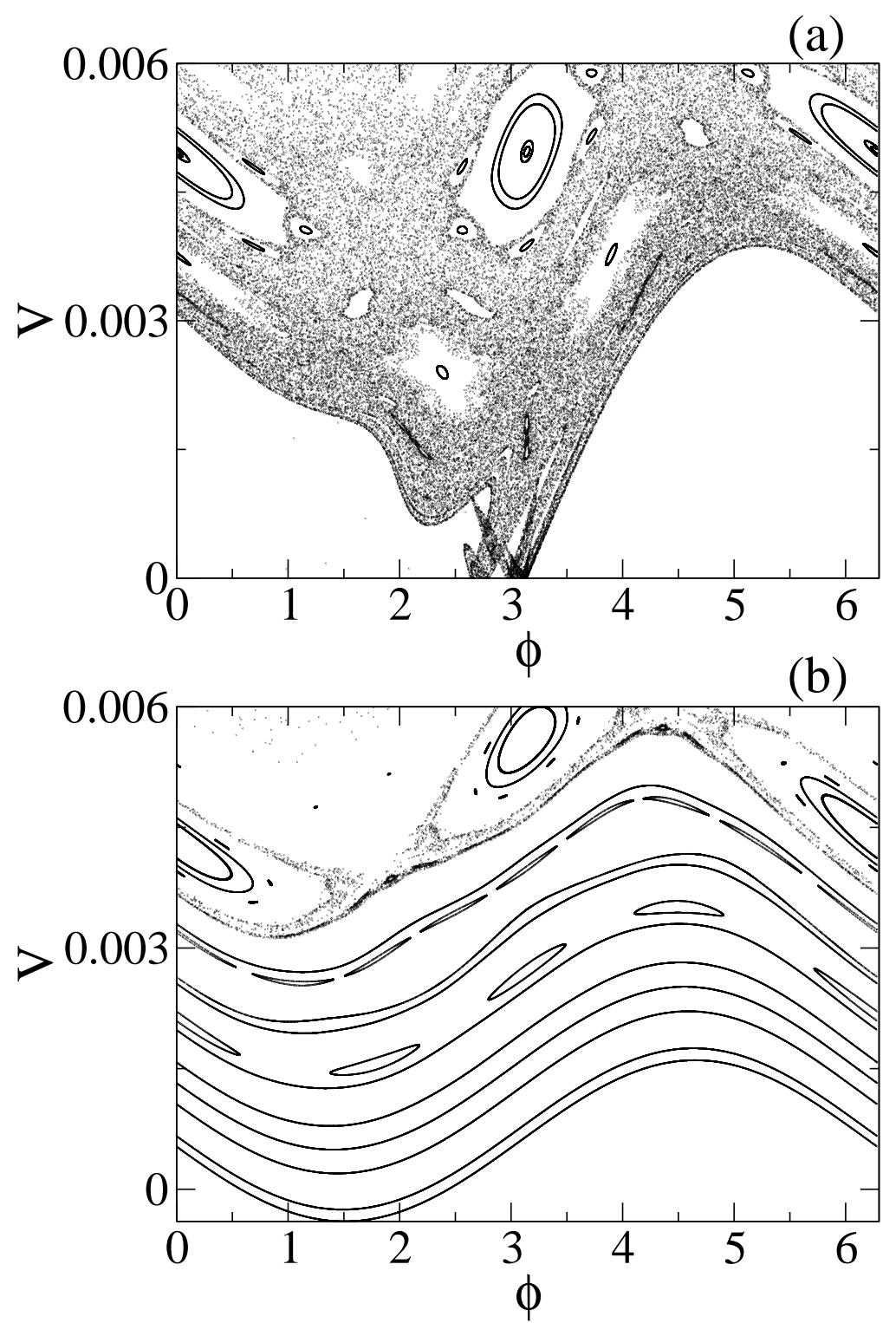

Figure 5. Expansion of Fig. 4 in the very low energy domain for both the (a) simplified and (b) complete models. The control parameters used here were $\epsilon=10^{-3}$ and $N_{c}=4$. (a) shows just positive velocities, and invariant spanning curves are not observed; (b) shows invariant spanning curves below the chaotic sea for the complete model.

sea may be observed for the complete version. Such curves give us clear evidence that the model is behaving as a bouncer. The basic condition of existence for such curves is, at least numerically, the same as that for the fixed point $\left(V_{n}, \phi_{n}\right)=\left(\frac{j}{2 \pi N_{c}^{2}}, 0\right)$ to be elliptic, in the simplified model, i.e. $\epsilon<\frac{1}{2 \pi^{2} N_{c}^{2}}$. Such curves appear to exist only in the complete model.

It was shown in Sec. 2.4 that the bouncer model undergoes a transition from locally to globally stochastic behaviour [39]. It means in our model that for $N_{c} \geq \frac{1}{2 \pi} \sqrt{\frac{0.971 \ldots}{2 \epsilon}}$ it is possible to observe a chaotic sea. However, we observe that the invariant spanning curves located below the chaotic sea exist when the condition $N_{c}<\frac{1}{\pi \sqrt{2 \epsilon}}$. In this sense, 
we can shall define a range for $N_{c}$ as function of $\epsilon$ in which these invariant spanning curves in the very low energy can coalesce with the chaotic sea. Such a range is given by $N_{c} \in\left[\frac{1}{2 \pi} \sqrt{\frac{0.971 \ldots}{2 \epsilon}}, \frac{1}{\pi \sqrt{2 \epsilon}}\right)$. Considering the parameter $\epsilon=10^{-3}$, the range of $N_{c}$ is $N_{c} \in[3.507 \ldots, 7.117 \ldots)$.

To illustrate the existence of these invariant spanning curves and the location of fixed points in the low energy domain, we construct the phase space for the following control parameters values $\epsilon=1 \times 10^{-3}$ and $N_{c}=4$, as shown in Fig. 4 . Note however that $N_{c}=4$ is in fact contained in the range obtained above. For easier comparison, we focus only on the chaotic low energy region for the simplified version, and the corresponding region for the complete version. An expansion of Fig. 4 in the very low energy domain is shown in Fig. 5. We can see that the complete and simplified versions behave quite differently in this energy limit. It is also clear that the complete model allows negative velocities, whereas they are of course not allowed in the simplified version. It is important to stress that the invariant spanning curves in the very low energy domain are not observed in the simplified version due to the properties of this version of the model in that energy limit. However they were foreseen to exist, and were then observed in the complete model.

It is interesting to note that our hybrid model has its own characteristics. In extreme ranges of control parameters, it recovers the results of the Fermi-Ulam model at high energy while exhibiting the results of the bouncer model in the low energy region for intense gravitational fields. Moreover, our results show that, within a certain range of control parameters, properties that are individually characteristic either of the Fermi-Ulam or of the bouncer model can come together and coexist in our hybrid model.

\section{Numerical results}

We discuss in this section our numerical results for the region of positive Lyapunov exponent in both the complete and simplified version of our model. We concentrate on the low energy chaotic region analysing the behaviour of the positive Lyapunov exponent $\lambda$ as function of $N_{c}$, as plotted in Fig. 6 with a fixed control parameter $\epsilon=10^{-3}$ for both (a) the complete and (b) the simplified models. Each point in this figure was obtained from the convergence of an ensemble average of 5 different initial conditions. In order to guarantee good convergence of the asymptotically positive Lyapunov exponent value, each initial condition was iterated $5 \times 10^{8}$ times. The error bars indicates the standard deviation of the five samples. We see in Fig. 6 that the Lyapunov exponent varies significantly as a function of $N_{c}$, and does so differently for the two versions. For the complete version, where we have a more realistic description of the problem, the positive Lyapunov exponent grows for small values of $N_{c}$ reaching a maximum and then decreasing asymptotically to a constant value. The maximum value of positive Lyapunov exponent is assumed to correspond to where the system experiences the strongest competition in whether to behave as a bouncer or as a Fermi-Ulam model. For the simplified version, it grows for small values of $N_{c}$ and reaches a regime of saturation 
(a)
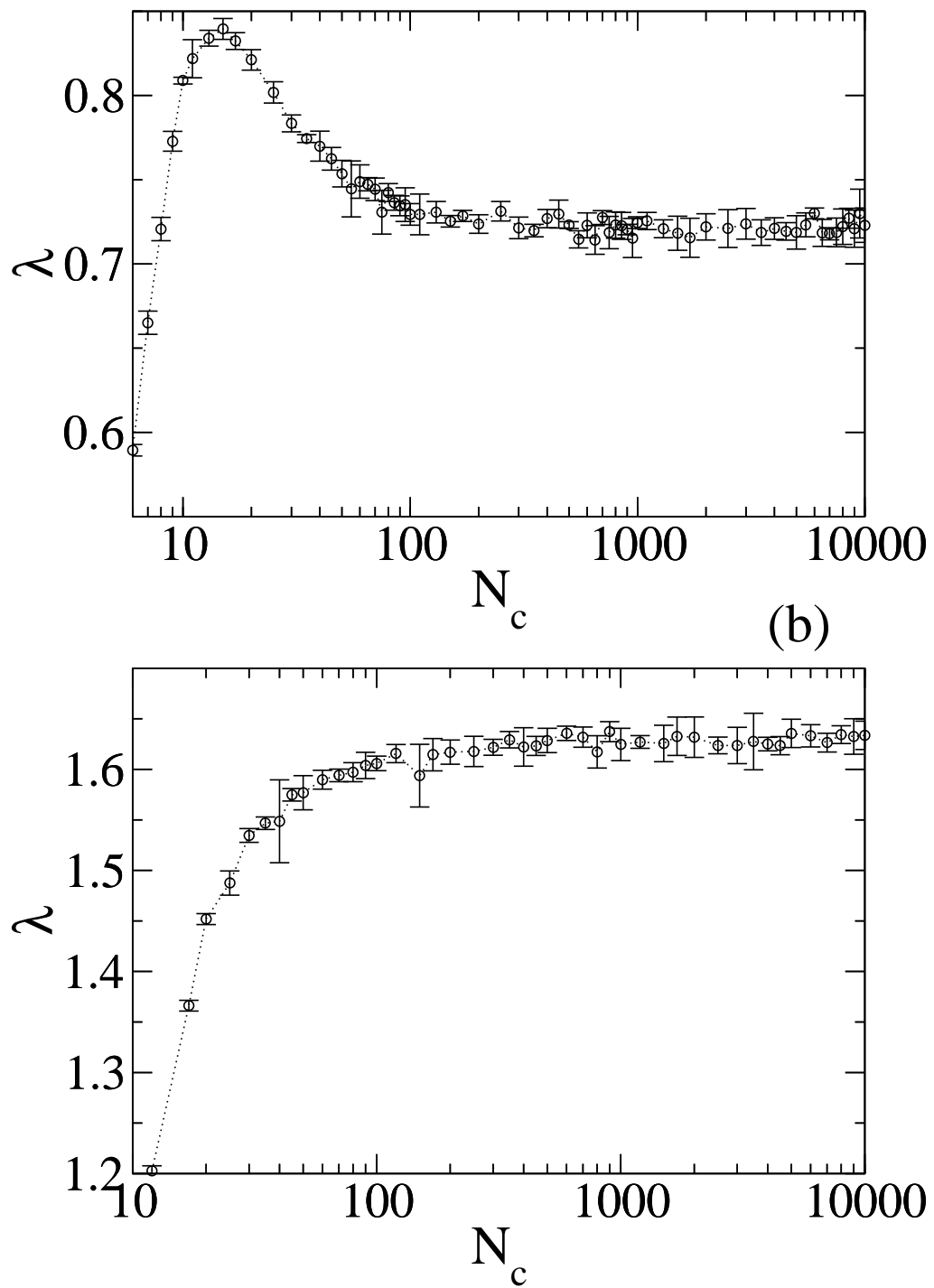

Figure 6. Log $\times$ linear graph illustrating the behaviour of the positive Lyapunov exponent as a function of $N_{c}$ for the control parameter $\epsilon=10^{-3}$ and the (a) complete and (b) simplified models.

for large values of $N_{c}$. Both the saturation and the convergence to a constant level can be explained as being due the fact of this model, in both its simplified and complete versions, must recover the Fermi-Ulam model results for very large $N_{c}$, as discussed in Sec. 3. In order to compare these asymptotic values with those from the Fermi-Ulam model, we evaluate the convergence of the positive Lyapunov exponent for this latter model in both complete and simplified versions. The Fermi-Ulam model is found to have $\lambda_{c}=0.722 \pm 0.008$ for its complete version, and $\lambda_{s}=1.63 \pm 0.01$ for its simplified one (see also Ref. [10] for Lyapunov exponents in the Fermi-Ulam model). Both results are in good agreement with those shown in Fig. 6. 
Now let us discuss why the positive Lyapunov exponent differs between the complete and simplified versions. Although the structure of fixed points, KAM islands and invariant spanning curves are very similar for both versions within their high energy domains, they are quite different in the low energy regimes. To illustrate one of these differences, we note that the complete version exhibits invariant spanning curves, whereas they are absent in the simplified version for $\epsilon<\frac{1}{2 \pi^{2} N_{c}^{2}}$. The obvious differences between the models are that: (i) the simplified model does not allow immediately sequential collisions; (ii) after the impact it does not allow a negative velocity; and (iii) it incorporates a forbidden region into the phase space.

\section{The stochastic model}

In this section we discuss some dynamical properties of the stochastic model. Our results for the complete and simplified deterministic models discussed above show that the phenomenon of Fermi acceleration does not occur for those versions. Its absence is attributable to the existence of invariant spanning curves in the phase space [11]. Such curves, in particular the first one above the chaotic sea, form boundaries limiting the size of the chaotic sea. In this sense, a particle evolving in the chaotic sea can never rise above the first invariant spanning curve. As we will see, the hierarchy of behaviours like KAM islands, chaotic sea, and invariant spanning curves does not arise in the stochastic model. The most interesting result for this version is that the velocity, and consequently the kinetic energy, grow as function of both iteration number $n$ and time $t$. The model is created by a subtle modification of the simplified version where (see above) a particle colliding with the wall located at $y=0$ exchanges momentum as if the wall was moving. In the stochastic model, such exchanges occur randomly. The map for this version is given by

$$
\left\{\begin{array}{l}
V_{n+1}=\left|V_{n}-2 \epsilon z\right| \\
t_{n+1}=t_{n}+\Delta T_{n}
\end{array}\right.
$$

where

$$
\Delta T_{n}=\left\{\begin{array}{lrc}
4 \pi^{2} N_{c}^{2}\left(V_{n}-\sqrt{V_{n}^{2}-\frac{1}{\pi^{2} N_{c}^{2}}}\right) & \text { if } \quad V_{n}>\frac{1}{\pi N_{c}} \\
4 \pi^{2} N_{c}^{2} V_{n} & \text { if } \quad V_{n} \leq \frac{1}{\pi N_{c}}
\end{array} .\right.
$$

Here, $z$ is a random number uniformly distributed in the interval $z \in[-1,1]$. Iteration of the stochastic map for one initial condition is shown in Fig. 7(a). The complex structure and hierarchy of the phase space present in the deterministic models are not observed here. Both KAM islands and invariant spanning curves are destroyed and, as a consequence of this, any one initial condition may lead to unlimited energy growth. To demonstrate this result we can compute the following observables

$$
\bar{V}=\frac{1}{M} \sum_{i=1}^{M}\left[\frac{1}{N} \sum_{n=1}^{N} V_{n, i}\right], \bar{t}=\frac{1}{M} \sum_{i=1}^{M}\left[\frac{1}{N} \sum_{n=1}^{N} t_{n, i}\right] .
$$

The sum over $N$ gives the average on the orbit, whereas the sum over $M$ gives the average over the ensemble of initial conditions and is just used to make the velocities 


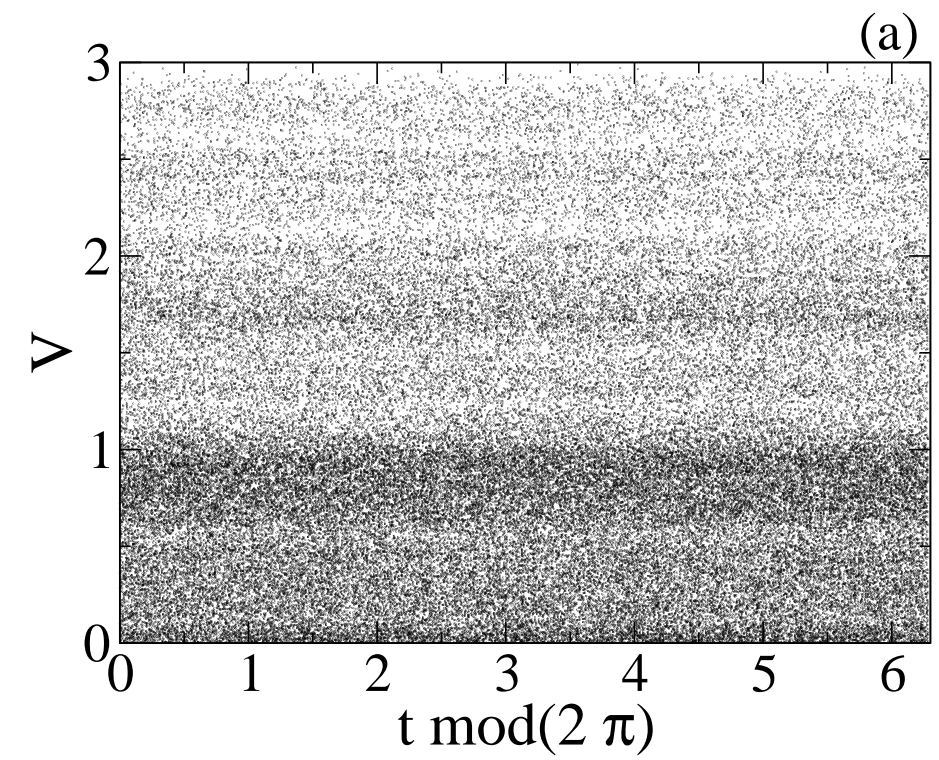

(b)

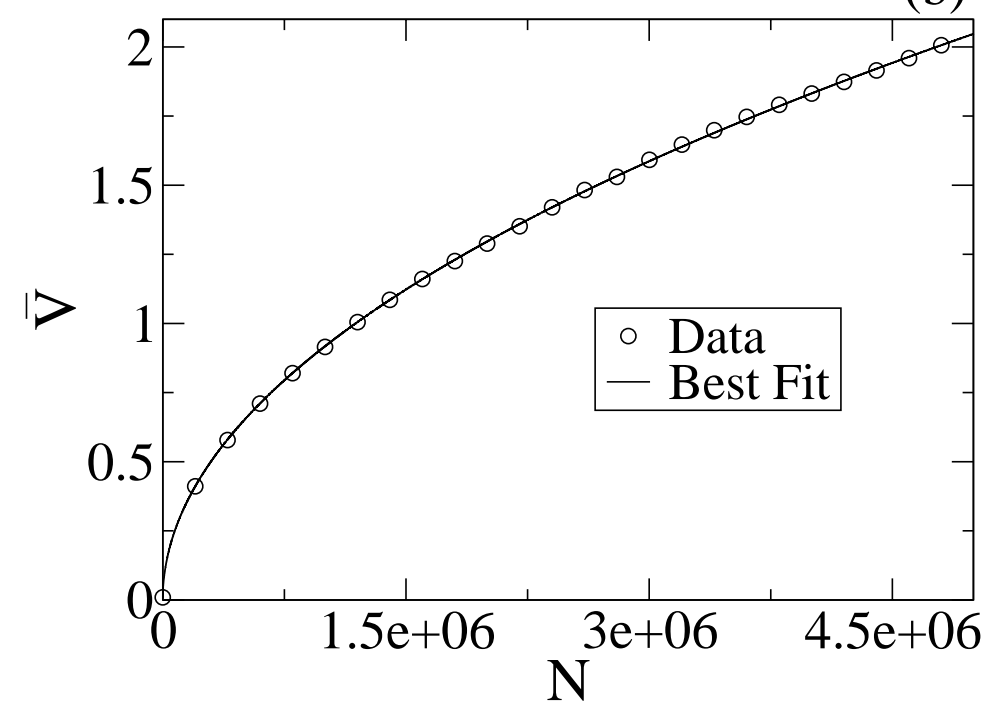

Figure 7. (a) Phase space for the stochastic model. (b) Behaviour of the average velocity as function of $N$. A power law fit yields $\bar{V} \propto N^{\delta_{n}}$ with $\delta_{n}=0.499(1)$. The control parameters used in both figures were $\epsilon=10^{-3}$ and $N_{c}=28$.

curves smoother and easier to characterise. Fig. 7(b) shows the behaviour of $\bar{V}$ as a function of $N ; \bar{V}$ as a function of $\bar{t}$ is shown in Fig. 8(a). The control parameters used for acquiring the data in these figures were $\epsilon=10^{-3}$ and $N_{c}=28$. We used an ensemble of 50,000 different initial conditions. All of them started with the same initial velocity $V_{0}=2 \times 10^{-3}$, and different seeds were used in the random number generator. Figs. 7(b) and 8(a) allow us to describe the behaviour of the average velocity as $\bar{V} \propto N^{\delta_{n}}$ and $\bar{V} \propto \bar{t}^{\delta_{t}}$. A power law fit gives $\delta_{n}=0.499(1)$ and this exponent is found to be the same for different control parameters. A power law fit for Fig. 8(a) gives the exponent $\delta_{t}=0.816(1)$. The exponent $\delta_{t}$ turns out to be dependent on $N_{c}$, as shown in Fig. 8(b) 

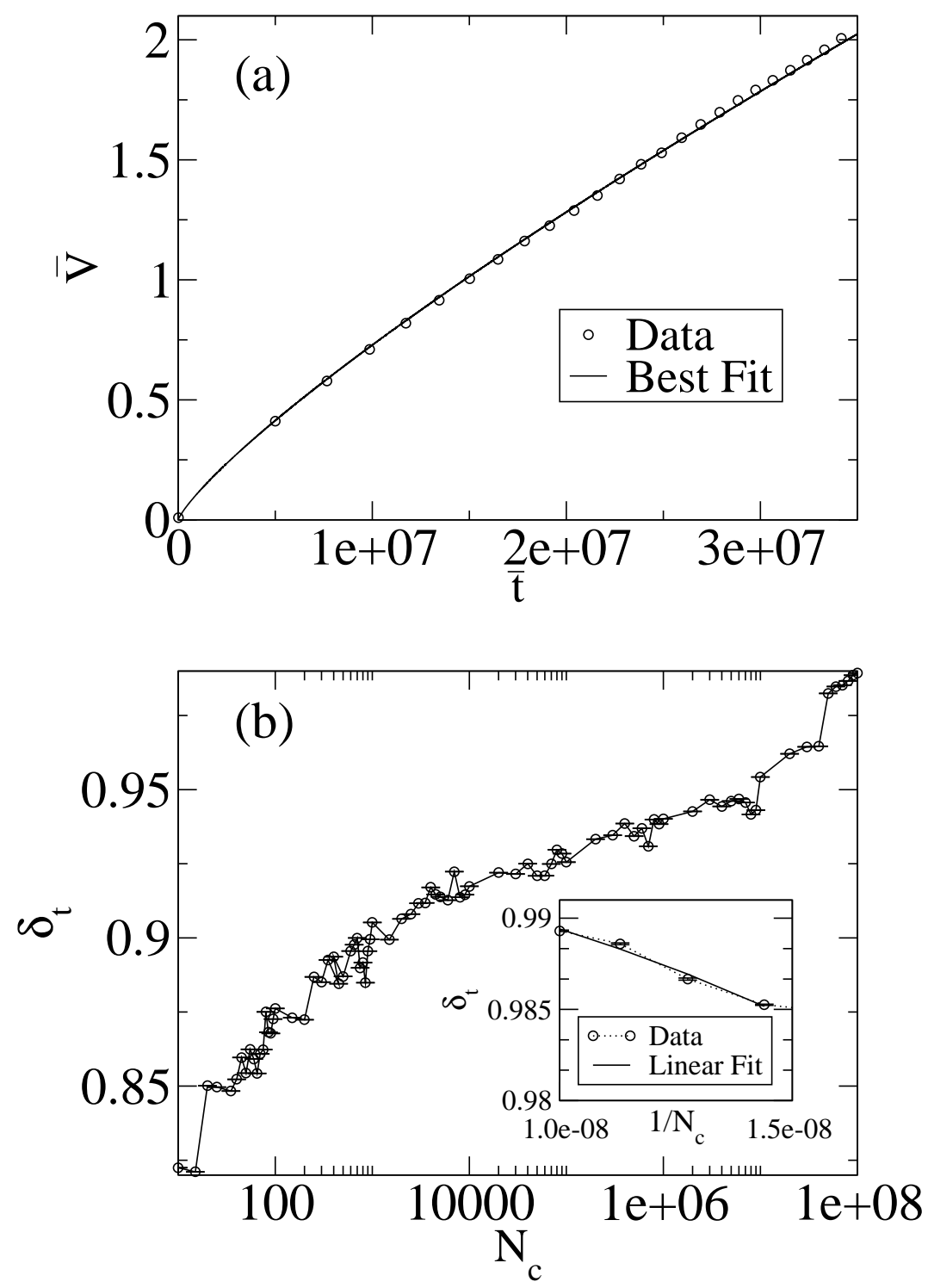

Figure 8. (a) Behaviour of the average velocity as function of time $t$. A power law fit suggests to us that $\bar{V} \propto \bar{t}^{\delta_{t}}$ with $\delta_{t}=0.816(1)$. The control parameters used here were $\epsilon=1 \times 10^{-3}$ and $N_{c}=28$. (b) Behaviour of $\delta_{t}$ as a function of $N_{c}$ for a fixed $\epsilon=10^{-3}$. The inset of (b) gives shows that $\delta_{t} \rightarrow 1$ when $1 / N_{c} \rightarrow 0$; the actual result obtained after extrapolation is $\delta_{t}=0.999(1)$.

which plots the variation of $\delta_{t}$ with $N_{c}$ for $\epsilon=10^{-3}$. The result for $N_{c} \rightarrow \infty$ indicates that $\delta_{t} \rightarrow 1$, as was found for the simplified stochastic Fermi-Ulam model [38]. It can be obtained by extrapolation, as shown in the inset of Fig. 8(b) after changing the horizontal coordinate to $N_{c} \rightarrow 1 / N_{c}$ and doing a linear fit. It is interesting to emphasise that the authors of [34] conjectured that Fermi acceleration should be observed for a billiard with a time-dependent boundary if the corresponding version for a fixed boundary presents chaotic components. However, we can conclude that for the system studied in the present paper (see also [25] and [26, 27] for comparable results in other systems) 
which exhibits chaotic behaviour under a time-dependent (periodic) perturbation, Fermi acceleration is observed only after the introduction of random (stochastic) motion to the time dependent wall.

\section{Summary and Conclusions}

We have studied a hybrid model behaving, not exclusively as a Fermi-Ulam model, nor as a bouncer, but as a combination between them. We analysed two different kind of movement: (i) periodic and (ii) random. For the periodic case, we derive two different versions of the system: complete; and simplified. We have shown that these systems present a rich and complex structure of phase space showing KAM islands, a chaotic sea and invariant spanning curves. We also show that these models, in both their complete and simplified forms, recover the results of the well known Fermi-Ulam model in the limit of zero external field. We show that the periodic orbits go over smoothly into the orbits of the Fermi-Ulam model. Such a transition characterises smooth evolution from one area-preserving map to another that preserves the same measure in the phase space. Considering the case of an intense external field and low energy regime, we observe that our model exhibits a similar structure of fixed points to that obtained in the bouncer model. In particular, the complete model presents the set of invariant spanning curves located below the chaotic sea that was foreseen by analysis of the simplified version of the model. We have also characterised the chaotic sea using Lyapunov exponents. Considering the case of random perturbation, we show that the structure of KAM islands and invariant spanning curves is completely destroyed and that, as a consequence, any initial condition in the phase space leads to Fermi acceleration. Such acceleration is characterised by unlimited velocity and correspondingly energy growth.

\section{Acknowledgements}

This research was supported by a grant from Conselho Nacional de Desenvolvimento Científico CNPq, from Brazil. The numerical results were obtained in the Centre for High Performance Computing in Lancaster University. The work was supported in part by the Engineering and Physical Sciences Research Council (UK).

\section{References}

[1] Fermi E 1949 Phys. Rev. 751169

[2] Walker G H and Ford J 1969 Phys. Rev. 188416

[3] Lichtenberg A J and Lieberman M A 1992 Regular and Chaotic Dynamics (Appl. Math. Sci. 38 Springer Verlag New York)

[4] Lieberman M A and Lichtenberg A J 1972 Phys. Rev. A 51852

[5] Kruger T, Pustylnikov L D and Troubetzkoy S E 1995 Nonlinearity 8397

[6] Wiesenfeld K and Tufillaro N B 1987 Physica D 26321

[7] Leonel E D, McClintock P V E and da Silva J K L 2004 Phys. Rev. Lett. 93014101

[8] Lieberman M A and Tsang K Y 1985 Phys. Rev. Lett. 55908 
[9] Tsang K Y and Ngai K L 1997 Phys. Rev. E 56 R17

[10] Leonel E D, da Silva J K L and Kamphorst S O 2004 Physica A 331435

[11] Ulam S 1961 Proceedings of the Fourth Berkeley Symposium on Math, Statistics and Probability Vol 3 (California: California University Press) p.315

[12] Pustylnikov L D 1983 Theor. Math. Phys. 57 1035; Pustylnikov L D 1987 Sov. Math. Dokl. 35(1) 88; Pustylnikov L D 1995 Russ. Acad. Sci. Sb. Math. 82(1)

[13] Lichtenberg A J, Lieberman M A and Cohen R H 1980 Physica D 1291

[14] Karner G 1994 J. Stat. Phys. 77867

[15] Dembinski S T, Makowski A J and Peplowski P 1993 Phys. Rev. Lett. 701093

[16] José J V and Cordery R 1986 Phys. Rev. Lett. 56290

[17] Seba P 1990 Phys. Rev. A 412306

[18] Jain S R 1993 Phys. Rev. Lett. 703553

[19] Kowalik Z J, Franaszek M and Pieranski P 1988 Phys. Rev. A 374016

[20] Warr S, Cooke W, Ball R C and Huntley J M 1996 Physica A 231551

[21] Warr S and Huntley J M 1995 Phys. Rev. E 525596

[22] Mateos J L and José J V 1998 Physica A 257434

[23] Mateos J L 1999 Phys. Lett. A 256113

[24] Luna-Acosta G A, Orellana-Rivadeneyra G, Mendoza-Galván A and Jung C 2001 Chaos, Solitons and Fractals 12349

[25] Leonel E D and da Silva J K L 2003 Physica A 323181

[26] Leonel E D and McClintock P V E 2004 Phys. Rev. E in press

[27] Leonel E D and McClintock P V E 2004 J. Phys. A: Math. Gen. submitted

[28] Luna-Acosta G A, Krokhin A A, Rodríguez M A, Hernández-Tejeda P H 1996 Phys. Rev. B 54 11410

[29] Luna-Acosta G A, Na K, Reichl L E and Krokhin A 1996 Phys. Rev. E 533271

[30] Luna-Acosta G A, Méndez-Bermúdez J A, Seba P and Pichugin K N 2002 Phys. Rev. E 65046605

[31] Berry M V 1981 Eur. J. Phys. 291

[32] Saitô N, Hirooka H, Ford J, Vivaldi F and Walker G H 1982 Physica D 5273

[33] Canale E, Markarian R, Kamphorst S O, de Carvalho S P 1998 Physica D 115189

[34] Loskutov A, Ryabov A B and Akinshin L G 2000 J. Phys. A: Math. Gen. 337973

[35] The simplified Fermi-Ulam model was introduced by Lichtenberg and Lieberman and can be found, for example in Ref. [3].

[36] Kamphorst S O and de Carvalho S P 1999 Nonlinearity 121363

[37] Eckmann J -P and Ruelle D 1985 Rev. Mod. Phys. 57617

[38] Leonel E D 2003 Chaotic Properties of Some Time Dependent Hamiltonian Systems. Ph.D. Thesis From Department of Physics, Federal University of Minas Gerais (Original version in Portuguese)

[39] We have used the same notation as the original work [3] although, at that time, stochasticity was frequently referred as to chaotic behaviour. However we emphasise that the transition is from locally to globally chaotic behaviour. In the Fermi-Ulam model, we mean by locally that the chaotic behaviour is confined by two different invariant spanning curves. 